\title{
Eosinophilic biomarkers for detection of acute exacerbation of chronic obstructive pulmonary disease with or without pulmonary embolism
}

\author{
QIONG-FANG YANG ${ }^{1}$, TING-TING LU ${ }^{2}$, CAI-MIN SHU ${ }^{1}$, LAN-FANG FENG $^{1}$, \\ HAO-TENG CHANG ${ }^{3,4}$ and QIAO-YING JI ${ }^{1}$
}

\begin{abstract}
Departments of ${ }^{1}$ Respiratory Medicine and ${ }^{2}$ Science Education, Affiliated Dongyang Hospital of Wenzhou Medical University, Dongyang, Zhejiang 322100, P.R. China; ${ }^{3}$ Graduate Institute of Basic Medical Science, China Medical University, Taichung $404 ;{ }^{4}$ Department of Computer Science and Information Engineering, Asia University, Taichung 413, Taiwan, R.O.C.
\end{abstract}

Received October 5, 2016; Accepted May 19, 2017

DOI: $10.3892 /$ etm.2017.4876

\begin{abstract}
Eosinophilia has been implicated in the pathophysiology of acute exacerbation of chronic obstructive pulmonary disease (AECOPD). However, the role of eosinophil activation in the development of AECOPD remains unclear. In the present study, the reliability of plasma levels of eosinophil activation markers, including eosinophil cationic protein (ECP), major basic protein (MBP), eosinophil-derived neurotoxin (EDN) and eosinophil peroxidase (EPX), were measured and used as diagnostic biomarkers of AECOPD with or without pulmonary embolism (PE). A total of 47 patients with AECOPD, 30 patients with AECOPD/PE and 35 healthy adults were enrolled in the present study. Plasma levels of ECP, EDN, EPX and MBP were measured using commercial ELISA kits. The mean concentrations of plasma ECP, EDN, EPX and MBP in the patients with AECOPD was significantly 2.87-, 3.06-, 1.60and 1.92-fold higher, respectively, compared with the control group $(\mathrm{P}<0.05)$. Similar results were obtained in patients with $\mathrm{AECOPD/PE}$, for whom plasma levels of ECP, EDN, EPX and MBP were significantly 2.06-, 2.21-, 1.42- and 2.42-fold higher, respectively, compared with the controls $(\mathrm{P}<0.05)$. No significant differences were observed in the levels of these proteins between patients with AECOPD or AECOPD/PE. Among the four potential markers, ECP was determined to be the optimal marker for distinguishing patients with AECOPD
\end{abstract}

Correspondence to: Dr Qiao-Ying Ji, Department of Respiratory Medicine, Affiliated Dongyang Hospital of Wenzhou Medical University, 60 Wuning West Road, Dongyang, Zhejiang 322100, P.R. China

E-mail: qyj195581@163.com

Key words: acute exacerbation of chronic obstructive pulmonary disease, pulmonary embolism, eosinophil cationic protein, eosinophil-derived neurotoxin, eosinophil peroxidase, major basic protein or AECOPD/PE from the controls. No significant correlation was observed between marker concentrations and gender, age or disease severity. The results of the present study may have clinical applications in the diagnosis of AECOPD using these novel biomarkers.

\section{Introduction}

Chronic obstructive pulmonary disease (COPD) is a chronic, progressive respiratory disease and, as the third largest cause of respiratory disease mortality, accounts for $23 \%$ of all respiratory disease mortalities (1). The diagnosis of COPD is changed to acute exacerbation of COPD (AECOPD) when respiratory symptoms worsen, beyond normal day-to-day variations, severely enough that changes in medication are required (2). AECOPD is associated with increased mortality and morbidity, and may have a profound impact on patients' quality of life (3). The causes of acute exacerbation include infectious agents, pneumothorax, myocardial infarction and pulmonary embolism (PE) $(2,4)$. The incidence of PE in patients with AECOPD is greater compared with those with stable COPD; for example, it is 5\% greater in South Korea (5), $18 \%$ greater in Israel (6) and 29.1\% greater in Turkey (7). COPD is an independent risk factor for PE (8), so once patients with AECOPD develop PE, respiratory and circulatory functions may deteriorate further, potentially becoming life threatening. Consistent with this observation, the mortality rate also significantly increases for patients with AECOPD/PE $(9,10)$. Thus, the early prediction and diagnosis for patients with AECOPD, with or without PE, is crucial.

AECOPD and PE are associated with a wide range of inflammatory cells, mediators and cytokine network components, including P-selectin $(11,12)$, inflammatory cytokines $(13,14)$ and leucocytes $(15,16)$. Previous studies have indicated that eosinophilic airway inflammation is also associated with the development of severe AECOPD (17-19). Blood eosinophilia has been reported to be associated with increased mortality in patients with COPD (20). When eosinophils are activated, four eosinophil secretory proteins are released systemically into 
body fluids; eosinophil cationic protein (ECP), major basic protein (MBP), eosinophil-derived neurotoxin (EDN) and eosinophil peroxidase (EPX) (21). ECP has various biological activities, including antibacterial, antiviral, antiparasitic and neurotoxic functions, and it contributes to the regulation of fibroblast activity (21). ECP also induces airway mucus secretion and interacts with the coagulation and complement systems $(22,23)$. Fiorini et al (24) concluded that serum ECP levels increased during AECOPD. Shahana et al (25) reported that MBP secreted by eosinophils induced the apoptosis of bronchial airway epithelial cells, leading to airway epithelial shedding and blister formation.

The role of eosinophil activation in the development of AECOPD with or without PE is unclear. Although ECP is increased in people with AECOPD (24), measuring this protein alone is insufficient to establish a correlation between eosinophil activation and AECOPD with or without PE. In the present study, the plasma levels of ECP, MBP, EPX and EDN were measured to assess and clarify the association between eosinophil activation and the development of AECOPD with or without PE. The plasma levels of these four proteins were measured in patients with AECOPD/PE, patients with AECOPD and control subjects. The results verified that eosinophils are activated in AECOPD; however, they were not significantly further activated in AECOPD/PE.

\section{Materials and methods}

Subjects. Demographic information for patients with AECOPD or AECOPD/PE and the normal control subjects is presented in Table I. A total of 30 patients with AECOPD/PE (male:female, 2:1), 47 patients with AECOPD (male:female, $30: 17$ ), and 35 age-matched healthy controls (male:female, 23:12) were recruited between April 2015 and December 2015 from the Affiliated Dongyang Hospital of Wenzhou Medical University (Dongyang, China). Informed consent was obtained from all participants prior to blood sampling, and all procedures were approved by the Institutional Review Board of the Affiliated Dongyang Hospital of Wenzhou Medical University. The age of the control subjects ranged from 62-87 years old (71.4 \pm 6.5 years); for patients with AECOPD/PE from 56-85 years old (72.2 \pm 7.7 years); and for patients with AECOPD from 57-89 years old (74.3 \pm 6.9 years). All patients were Han Chinese. The diagnosis of COPD was confirmed based on medical history, medical records and available spirometric data. The clinical severity of COPD was determined using the criteria of the Global Initiative for Chronic Obstructive Lung Disease (GOLD) (26). The clinical conditions of the patients with AECOPD and AECOPD/PE were evaluated on the same day that blood samples were taken, and patients were accordingly classified into one of four GOLD subindices (GOLD1-4). Acute exacerbation was diagnosed when respiratory symptoms in patients with COPD worsened to the point where medication change was required. The diagnosis of PE was based on a computed tomography scans, which were performed after the patients' written informed consent was received and on the same day as blood sampling, and was defined by a sharply delineated pulmonary arterial filling defect present in at least two consecutive image sections and located centrally within the vessel, or with acute angles at its interface with the vessel wall. Upon arrival at the hospital, Emergency or Respiratory Department physicians evaluated all patients by taking their history and assessing the results of their physical examinations, chest radiographs and several routine laboratory tests, including routine blood testing to examine the blood chemistry and coagulation function. Patients with any specific issues identified in those examinations, including pneumothorax, congestive heart failure, cancer of any kind or a history of major surgery in the preceding 4 weeks were excluded from the present study. Patients undergoing mechanical ventilation, receiving anticoagulation therapy for any cause, presenting with azotemia (serum creatinine $>1.5 \mathrm{mg} / \mathrm{dl}$ ) or with a hypersensitivity reaction to contrast media were also excluded. Following exclusion, all remaining patients underwent computed tomography pulmonary angiography and indirect computed tomography venography within $24 \mathrm{~h}$ of admission to the hospital. All patients enrolled in the present study were given regular treatments, including antibiotics, bronchodilators, inhaled glucocorticoid hormones, expectorants and other conventional therapies at regular dosages.

Blood sampling and laboratory assays. Venous blood $(10 \mathrm{ml})$ was collected from each participant into lithium heparin tubes. Plasma was separated by centrifugation at 3,000 $\mathrm{x}$ g for $10 \mathrm{~min}$ at $4^{\circ} \mathrm{C}$, aliquoted and stored at $-80^{\circ} \mathrm{C}$ until required. Peripheral blood counts were examined for leukocytes, eosinophils, neutrophils and lymphocytes, and the plasma concentrations of ECP, MBP, EPX, EDN, low density lipoprotein, high density lipoprotein, triglycerides, total cholesterol and albumin. ELISA kits were used to measure the concentrations of plasma ECP (SK00128-01; Aviscera Bioscience, Inc., Santa Clara, CA, USA), EDN (CODE7630; Medical and Biological Laboratories Co., Ltd., Nagoya, Japan), EPX (SEJ138Hu; Cloud-Clone Corp., Katy, TX, USA) and MBP (SEB650Hu; Cloud-Clone Corp.).

Statistical analysis. All data were analyzed using GraphPad Prism software (version 5.0; GraphPad Software, Inc., La Jolla, CA, USA). The plasma concentrations of ECP, EDN, EPX and MBP among patients in the AECOPD, AECOPD/PE and control groups were compared using one-way analysis of variance with a post hoc Bonferroni test to determine the statistical significance of the differences between groups. The correlation between each GOLD index and ECP, EDN, EPX and MBP concentration was calculated using the Pearson correlation coefficient. The diagnostic accuracies of the ECP, EDN, EPX and MBP concentrations were analyzed using a receiver-operating characteristic (ROC) curve that was constructed by plotting the true-positive proportion (sensitivity) vs. the false-positive proportion (1-specificity) for each cut-off value. Thus, each ROC curve displayed the trade-off between sensitivity and specificity for all possible cut-offs. Diagnostic performance was measured as the area under a ROC curve. $\mathrm{P}<0.05$ was considered to indicate a statistically significant difference.

\section{Results}

Patient population. A total of 112 patients [age, 73.66 7.46 ; male, $n=73(65 \%)]$ were enrolled in the present study. Of these, 
Table I. Clinicopathological characteristics of the patient groups.

\begin{tabular}{lcccc}
\hline Variable & Control $(\mathrm{n}=35)$ & AECOPD $(\mathrm{n}=47)$ & AECOPD/PE $(\mathrm{n}=30)$ & P-value \\
\hline Age, years & $71.40 \pm 6.50$ & $74.30 \pm 6.90$ & $72.20 \pm 7.70$ & 0.153 \\
Male, $\mathrm{n}(\%)$ & $23(66)$ & $30(64)$ & $20(67)$ & 0.9647 \\
Diabetes, $\mathrm{n}(\%)$ & $4(11)$ & $6(13)$ & $5(17)$ & 0.8148 \\
Hypertension, $\mathrm{n}(\%)$ & $11(31)$ & $16(34)$ & $8(27)$ & 0.2625 \\
Coronary disease, $\mathrm{n}(\%)$ & $0(0)$ & $2(4)$ & $8(27)^{\mathrm{c}}$ & 0.0003 \\
AF, $\mathrm{n}(\%)$ & $0(0)$ & $0(0)$ & $3(10)$ & 0.0148 \\
CTD, $\mathrm{n}(\%)$ & $0(0)$ & $2(4)$ & $5(17)^{\mathrm{c}}$ & 0.0165 \\
WBC count $\left(\mathrm{x} 10^{9} / \mathrm{l}\right)$ & $5.40 \pm 1.40$ & $8.40 \pm 5.70$ & $9.50 \pm 3.30^{\mathrm{c}}$ & 0.090 \\
Eosinophil count $\left(\mathrm{x} 10^{9} / \mathrm{l}\right)$ & $0.10 \pm 0.06$ & $0.11 \pm 0.12$ & $0.12 \pm 0.12$ & 0.799 \\
Neutrophil count $\left(\mathrm{x} 10^{9} / \mathrm{l}\right)$ & $3.49 \pm 0.97^{\mathrm{a}}$ & $7.09 \pm 0.82^{\mathrm{b}}$ & $8.43 \pm 0.61^{\mathrm{c}}$ & 0.001 \\
Lymphocyte count $\left(\mathrm{x} 10^{9} / \mathrm{l}\right)$ & $1.43 \pm 0.46$ & $1.84 \pm 0.68^{\mathrm{b}}$ & $1.26 \pm 0.67$ & 0.001 \\
LDL (mg/dl) & $2.91 \pm 1.12^{\mathrm{a}}$ & $2.30 \pm 0.85$ & $2.27 \pm 0.62^{\mathrm{c}}$ & 0.010 \\
HDL (mg/dl) & $1.40 \pm 0.27^{\mathrm{a}}$ & $1.12 \pm 0.36$ & $1.12 \pm 0.37^{\mathrm{c}}$ & 0.003 \\
TG (mg/dl) & $1.58 \pm 0.77^{\mathrm{a}}$ & $1.04 \pm 0.41$ & $1.09 \pm 0.44^{\mathrm{c}}$ & 0.001 \\
TC (mg/dl) & $6.54 \pm 7.94^{\mathrm{a}}$ & $4.04 \pm 1.06$ & $4.05 \pm 0.72$ & 0.031 \\
Albumin $(\mathrm{g} / \mathrm{l})$ & $46.67 \pm 2.56^{\mathrm{a}}$ & $36.44 \pm 8.59$ & $37.44 \pm 3.48^{\mathrm{c}}$ & $<0.001$ \\
\hline
\end{tabular}

${ }^{\mathrm{a}} \mathrm{P}<0.05$ vs. AECOPD group; ${ }^{\mathrm{P}}<0.05$ vs. AECOPD/PE group; ${ }^{\mathrm{P}}<0.05$ vs. AECOPD/PE group. The determined $\mathrm{P}$-value for each variable in the table were comparisons between all three groups. AECOPD, acute exacerbation of chronic obstructive pulmonary disease; PE, pulmonary embolism; AF, atrial fibrillation; CTD, connective tissue disease; WBC, white blood cell; LDL, low-density lipoprotein; HDL, high-density lipoprotein; TG, triglycerides; TC, total cholesterol.

77 (69\%) patients underwent computed tomography pulmonary angiography and 30 were diagnosed with PE (39\%). Baseline characteristics for the three groups are listed in Table I. No significant difference was observed in age or gender; however, significant differences were identified in PE risk factors, including atrial fibrillation and connective tissue disease, which were significantly more frequent in patients with AECOPD/PE compared with the other two groups $(\mathrm{P}<0.05)$. Furthermore, patients with AECOPD/PE or AECOPD had significantly lower blood lipid, apolipoprotein and albumin levels $(\mathrm{P}<0.05)$, suggesting that these patients had a relatively poor nutritional status. Leukocyte counts in patients with AECOPD/PE were significantly higher compared with those of the controls and patients with AECOPD $(\mathrm{P}<0.05)$; neutrophil counts displayed the same trend when comparing all 3 groups $(\mathrm{P}<0.001)$; similarly, the difference between AECOPD/PE and AECOPD was significant $(\mathrm{P}<0.05)$.

Comparison of plasma levels of ECP, EDN, EPX and MBP. In patients with AECOPD/PE, the mean concentrations of plasma ECP, EDN, EPX and MBP were 12.40 \pm 8.29 and $52.72 \pm 30.77 \mathrm{ng} / \mathrm{ml}$, and $3.82 \pm 2.47$ and $1.74 \pm 0.83 \mu \mathrm{g} / \mathrm{ml}$, respectively (Fig. 1). ECP $(\mathrm{P}<0.001)$, EDN $(\mathrm{P}<0.001)$, EPX $(\mathrm{P}<0.05)$ and MBP $(\mathrm{P}<0.001)$ levels were significantly greater in patients with AECOPD/PE compared with the corresponding values for the healthy controls $(6.02 \pm 2.30$ and $23.90 \pm 10.94 \mathrm{ng} / \mathrm{ml}$, and $2.69 \pm 1.71$ and $0.72 \pm 0.27 \mu \mathrm{g} / \mathrm{ml}$, respectively; Fig. 1); however, no significant difference was observed in these levels between patients with AECOPD/PE and patients with AECOPD $(17.26 \pm 12.90$ and $73.12 \pm 55.19 \mathrm{ng} / \mathrm{ml}$, and $4.31 \pm 2.76$ and $1.38 \pm 0.72 \mu \mathrm{g} / \mathrm{ml}$, respectively). Similarly, the concentrations of plasma ECP $(\mathrm{P}<0.001)$, EDN $(\mathrm{P}<0.001)$, EPX $(\mathrm{P}<0.01)$ and MBP $(\mathrm{P}<0.001)$ were significantly greater in patients with AECOPD compared with the control group. These results suggest that ECP, EDN, EPX and MBP plasma concentrations may serve as biomarkers for the diagnosis of AECOPD with or without PE; however, a full evaluation of their effectiveness is required, including further comparisons involving other chronic inflammatory diseases that clinically resemble AECOPD.

Predictive performance of ECP, EDN, EPX and MBP for $A E C O P D$. ROC curve analysis was employed to determine the performance of ECP,EDN, EPX and MBP as biomarkers for the diagnosis of AECOPD or AECOPD/PE. Cut-off values were used to distinguish patients with AECOPD or AECOPD/PE from the healthy controls, and the associated biomarkers for their performance were assayed by measuring the area under the relevant ROC curve (Fig. 2). In these analyses, using an AECOPD:control (AEC:c) cut-off of $6.98 \mathrm{ng} / \mathrm{ml}$, ECP exhibited the best performance with an accuracy, sensitivity and specificity of 80.50, 57.45 and $97.14 \%$, respectively, and an AUC of 0.8328 (Fig. 2A). Similarly, with an AECOPD/PE:control (AEC/PE:c) cut-off of $7.78 \mathrm{ng} / \mathrm{ml}$, ECP had an accuracy, sensitivity and specificity of $81.50,46.67$ and $97.14 \%$, respectively, with an AUC of 0.7905 (Fig. 2A). No significant difference was observed between patients with AECOPD and patients with AECOPD/PE.

With an AEC:c cut-off of $33.31 \mathrm{ng} / \mathrm{ml}$, EDN had $78.00 \%$ accuracy, $61.70 \%$ sensitivity and $100.00 \%$ specificity with an AUC value of 0.7954 (Fig. 2B). With an AEC/PE:c cut-off of $37.62 \mathrm{ng}$, EDN had $78.50 \%$ accuracy, $56.67 \%$ sensitivity and 


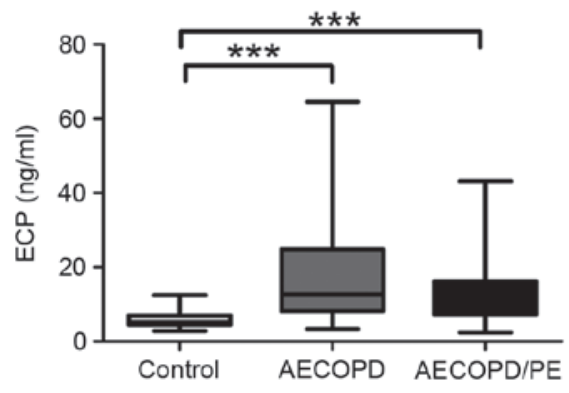

C

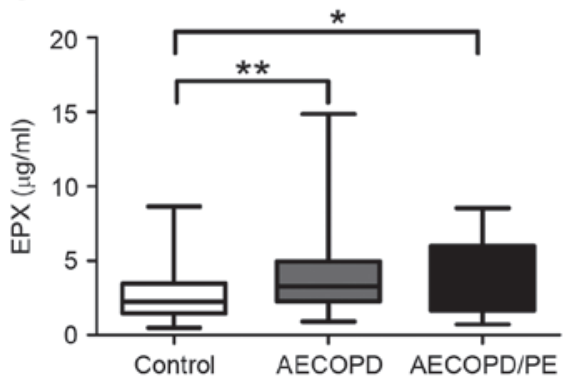

B

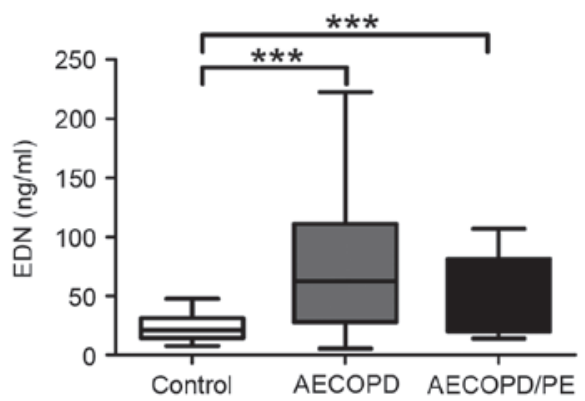

D

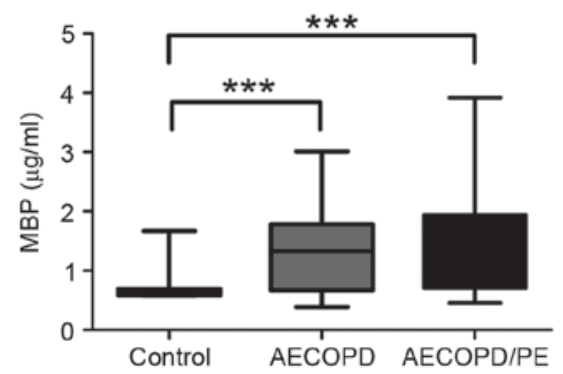

Figure 1. Plasma levels of (A) ECP $\left({ }^{* * *} \mathrm{P}<0.001\right.$ vs. AECOPD and AECOPD/PE at ECP), (B) EDN $\left({ }^{* * *} \mathrm{P}<0.001\right.$ vs. AECOPD and AECOPD/PE at EDN), (C) EPX ("P<0.05 vs. AECOPD/PE at EPX; ${ }^{* *} \mathrm{P}<0.01$ vs. AECOPD at EPX) and (D) MBP in normal controls ( $\left.\mathrm{n}=35\right)$, patients with AECOPD/PE (n=30) and patients with AECOPD $(n=47)\left({ }^{* * *} \mathrm{P}<0.001\right.$ vs. AECOPD and AECOPD/PE at MBP). The edges of the boxes represent the interquartile ranges, and the solid lines drawn across the boxes represent the medians. ECP, eosinophil cationic protein; EDN, eosinophil-derived neurotoxin; EPX, eosinophil peroxidase; MBP, major basic protein; AECOPD, acute exacerbation of chronic obstructive pulmonary disease; PE, pulmonary embolism.

A

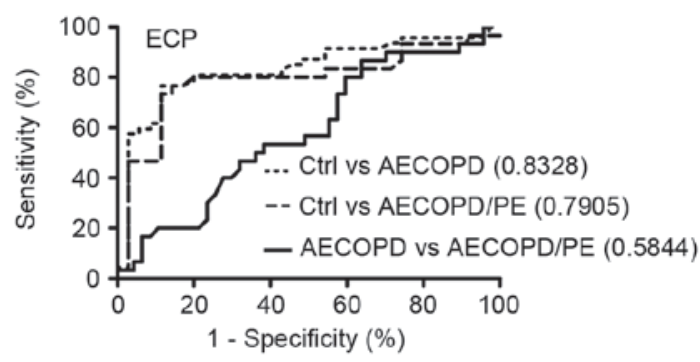

C

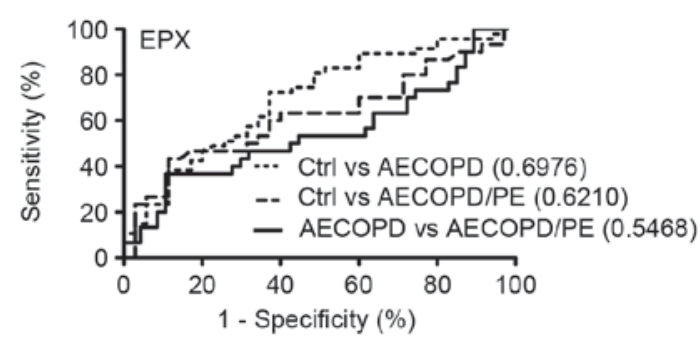

B

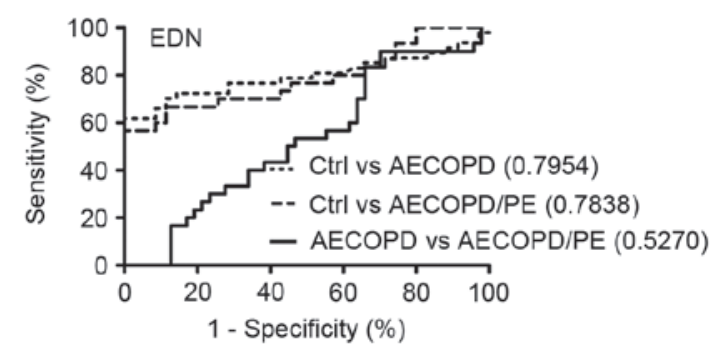

D

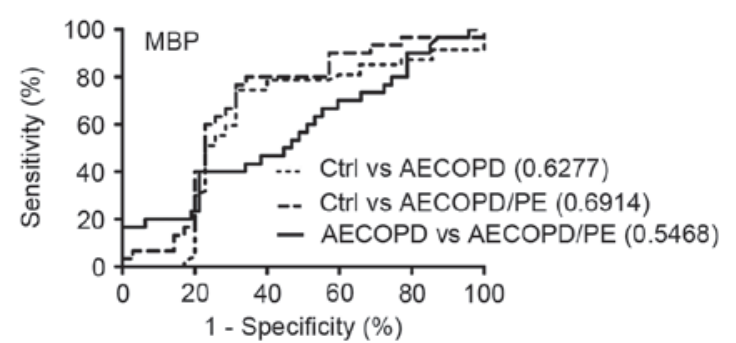

Figure 2. ROC curves for (A) ECP, (B) EDN, (C) EPX and (D) MBP in the diagnosis of AECOPD with or without PE. The ROC curves illustrate the comparison among patients with AECOPD/PE $(n=30)$, patients with AECOPD $(n=47)$ and normal controls $(n=35)$. The area under each curve is shown in parentheses. ROC, receiver-operating characteristic; ECP, eosinophil cationic protein; EDN, eosinophil-derived neurotoxin; EPX, eosinophil peroxidase; MBP, major basic protein; AECOPD, acute exacerbation of chronic obstructive pulmonary disease; PE, pulmonary embolism; Ctrl, control.

$100.00 \%$ specificity with an AUC of 0.7838 (Fig. 2B). With an AEC:c cut-off of $2.24 \mu \mathrm{g} / \mathrm{ml}$, EPX had $68.30 \%$ accuracy, $14.89 \%$ sensitivity and $97.14 \%$ specificity with an AUC of
0.6976; and with an AEC/PE:c cut-off of $4.07 \mu \mathrm{g} / \mathrm{ml}$, EPX had $67.70 \%$ accuracy, $23.33 \%$ sensitivity and $97.14 \%$ specificity with an AUC of 0.6210 (Fig. 2C). Finally, with an AEC:c 
A

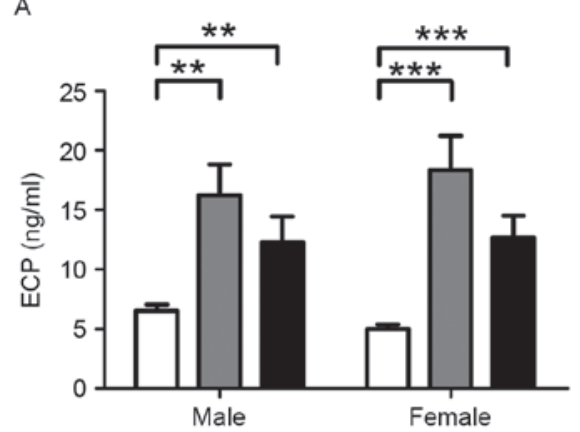

C

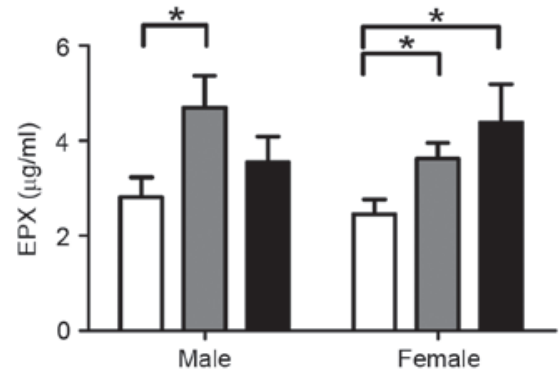

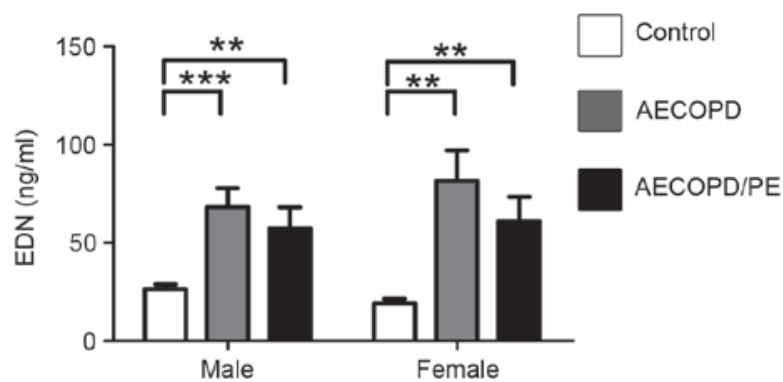

D

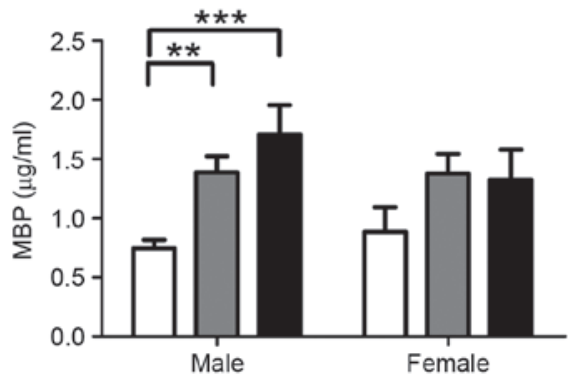

Figure 3. Levels of (A) ECP ("P $<0.01$ vs. AECOPD and AECOP/PE at ECP in male; ${ }^{* * *} \mathrm{P}<0.001$ vs. AECOPD and AECOPD/PE at ECP in female), (B) EDN $\left({ }^{* *} \mathrm{P}<0.01\right.$ vs. $\mathrm{AECOPD} / \mathrm{PE}$ at EDN in male, and vs. AECOPD and AECOPD/PE at EDN in female; ${ }^{* * *} \mathrm{P}<0.001$ vs. AECOPD at EDN in male), (C) EPX $\left({ }^{*} \mathrm{P}<0.05\right.$ vs. AECOPD at EPX in male, and vs. AECOPD and AECOPD/PE at EPX in female) and (D) MBP classified by sex in normal controls and patients with AECOPD/PE or AECOPD $\left({ }^{* *} \mathrm{P}<0.01\right.$ vs. AECOPD at MBP in male; ${ }^{* * *} \mathrm{P}<0.001$ vs. AECOPD/PE at MBP in male). Normal controls (males, 23 ; females, 12); patients with AECOPD (males, 30; females, 17); patients with AECOPD/PE, (males, 20; females, 10). ECP, eosinophil cationic protein; EDN, eosinophil-derived neurotoxin; EPX, eosinophil peroxidase; MBP, major basic protein; AECOPD, acute exacerbation of chronic obstructive pulmonary disease; PE, pulmonary embolism.

cut-off of $0.77 \mu \mathrm{g} / \mathrm{ml}$, MBP had $72.00 \%$ accuracy, $74.47 \%$ sensitivity and $68.57 \%$ specificity with an AUC of 0.6277 ; and with an AEC/PE:c cut-off value of $0.73 \mu \mathrm{g} / \mathrm{ml}$, MBP had $72.30 \%$ accuracy, $76.67 \%$ sensitivity and $68.57 \%$ specificity with an AUC of 0.6914 (Fig. 2D). These results indicate that plasma levels of ECP, EDN, EPX and MBP are useful indicators for distinguishing patients with AECOPD or AECOPD/PE from healthy controls.

Comparison of ECP, EDN, EPX, and MBP across AECOPD and $A E C O P D / P E$ patient characteristics. The patients in the AECOPD, AECOPD/PE and control groups were categorized based on gender, age and disease severity for further statistical analysis. Patient characteristics that correlated with increased levels of ECP, EDN, EPX and MBP were examined. When corrected for sex, the concentrations of all proteins were greater in the disease groups ( $\mathrm{P}<0.05$ vs. the control group; Fig. 3$)$, with the exception of MBP in females (Fig. 3D). When corrected for age, the concentrations of ECP, EDN and MBP were significantly higher in the disease groups in the 55-69 and 70-79 years age brackets ( $\mathrm{P}<0.05$ vs. the control group; Fig. 4). Protein levels were not significantly different between groups in the 80-89-year age bracket (Fig. 4); however, this may be because there were so few cases in this age bracket in the control group.

Fig. 5 indicated That the plasma levels of ECP and EDN were significantly increased in the disease subgroups, as defined by the GOLD indices, compared with the controls $(\mathrm{P}<0.01$; Fig. $5 \mathrm{~A}, \mathrm{~B}, \mathrm{E}$ and $\mathrm{F})$. The levels of EPX were significantly increased in the AECOPD group in GOLD 2
$(\mathrm{P}<0.001), 3(\mathrm{P}<0.05)$ and $4(\mathrm{P}<0.05)$, and the AECOPD/PE group in GOLD 2 and 3 (both $\mathrm{P}<0.05$ ) compared with the controls (Fig. 5C and G). The concentration of MBP was just higher in AECOPD of GOLD $2(\mathrm{P}<0.01)$ and $3(\mathrm{P}<0.05)$, and AECOPD/PE of GOLD 3 (P<0.05; Fig. 5D and H). No significant correlation was observed between the protein levels and disease severity.

\section{Discussion}

The present study demonstrated that the plasma levels of ECP, EDN, EPX, and MBP, secreted from activated eosinophil granulocytes, were significantly higher in patients with AECOPD or AECOPD/PE compared with healthy controls, suggesting that eosinophil activation is associated with AECOPD with or without PE. However, no significant differences in the plasma levels of eosinophil activation markers were observed between the AECOPD and AECOPD/PE groups. This suggests that the activation of eosinophils has a greater impact on the development of AECOPD in healthy subjects compared with the development of PE in existing AECOPD. To the best of our knowledge, this is the first study to examine the association between eosinophil secretory proteins and AECOPD with or without PE.

A number of studies have addressed the association between neutrophils (27), eosinophils (18), T lymphocytes (28) and macrophages (29) and the pathogenesis of AECOPD. The factors that lead to the development of AECOPD from COPD remain unclear. Smoking, air 
A
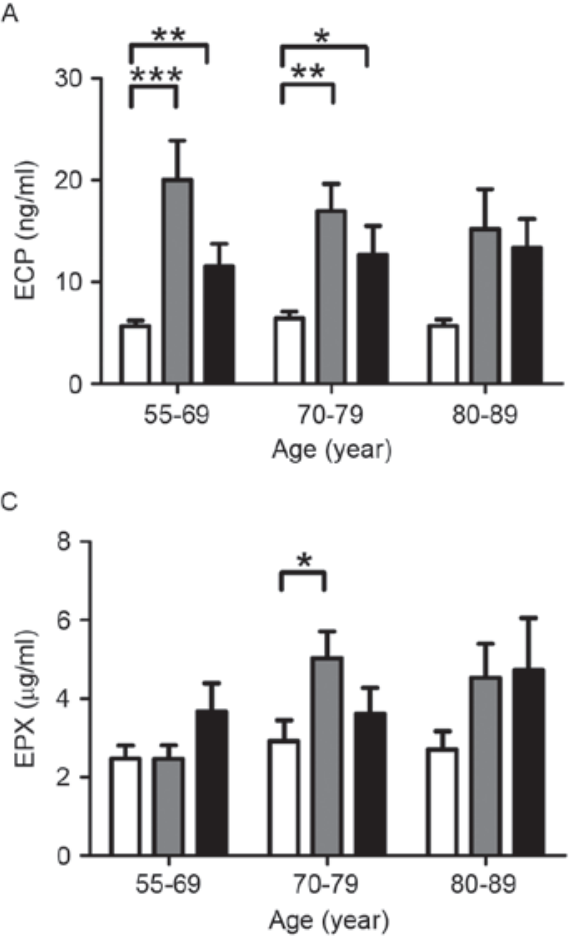

B

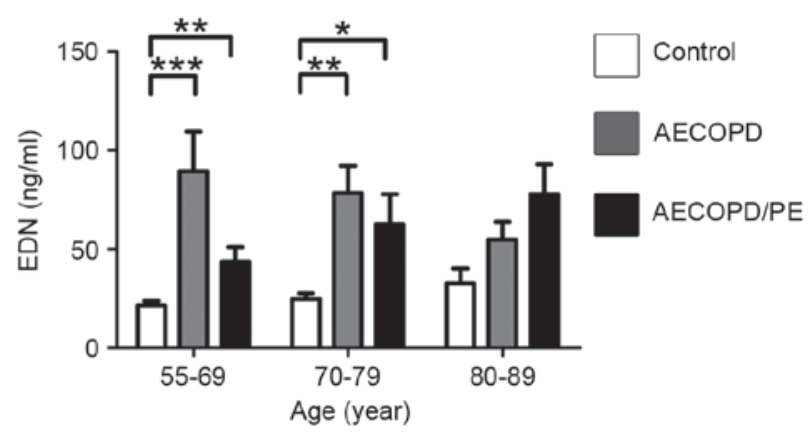

$\mathrm{D}$

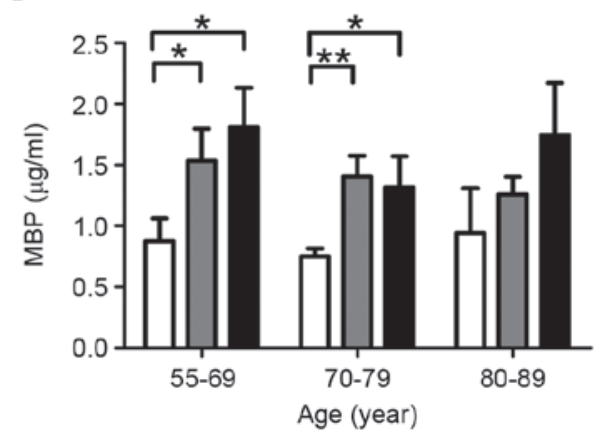

Figure 4.Levels of (A) ECP ("P $<0.05$ vs. AECOPD/PE at ECP in the 70-79 age group; ${ }^{* *} \mathrm{P}<0.01$ vs. AECOPD/PE at ECP in the 55-69 age group and AECOPD at ECP in the 70-79 age group; ${ }^{* * *} \mathrm{P}<0.001$ vs. AECOPD at ECP in the 55-69 age group), (B) EDN ("P<0.05 vs. AECOPD/PE at EDN in the $70-79$ age group; ${ }^{* *} \mathrm{P}<0.01$ vs. AECOPD/PEatEDN in the 55-69 age group and AECOPD atEDN in the 70-79 age group; ${ }^{* * *} \mathrm{P}<0.001$ vs. AECOPD atEDN in the 55-69 age group), (C) EPX ("P<0.05 vs.AECOPDatEPXinthe 70-79agegroup), and(D) MBPclassified by ageinnormalcontrolsand patients with AECOPD/PEorwith AECOPD("P $<0.05$ vs.AECOPD/ PEat MBPin the 55-69 and 70-79 age groups, and vs. AECOPD at MBP in the 55-69 age group; ${ }^{* *} \mathrm{P}<0.01 \mathrm{vs}$. AECOPD at MBP in the 70-79 age group). Normal controls (ages 55-69, $\mathrm{n}=16$; ages 70-79, $\mathrm{n}=16$; ages 80-89, $\mathrm{n}=3$ ); patients with AECOPD (ages 55-69, $\mathrm{n}=10$; ages 70-79, $\mathrm{n}=21$; ages 80-89, $\mathrm{n}=16$ ); patients with AECOPD/PE (ages 55-69, n=12; ages 70-79, n=13; ages 80-89, n=5). ECP, eosinophil cationic protein; EDN, eosinophil-derived neurotoxin; EPX, eosinophil peroxidase; MBP, major basic protein; AECOPD, acute exacerbation of chronic obstructive pulmonary disease; PE, pulmonary embolism.

pollution, allergen inhalation, surgery, sedative drug use and infections have been reported to be correlated with the development of AECOPD (30). Clinical investigations have reported that eosinophils are increased in the sputum and bronchial biopsies of patients with AECOPD $(31,32)$. Increased levels of ECP in serum $(24,33)$, bronchoalveolar lavage fluid (34) and induced sputum (35) have been observed in patients with AECOPD. Hospers et al (20) proposed that peripheral blood eosinophilia may also lead to a higher mortality rate for patients with AECOPD. Fujimoto et al (36) further confirmed that the ECP level significantly increases in the transition from stable COPD to AECOPD. The results of the present study identified an increased leukocyte count and higher plasma levels of ECP, EDN, EPX and MBP in patients with AECOPD compared with the control group. These results suggest that during the exacerbation stage of COPD, eosinophils are activated and degranulated, and that the eosinophil secretory proteins ECP, EDN, EPX and MBP are released systemically into body fluids. Furthermore, these proteins have cytotoxic effects that damage airway epithelial cells, induce the secretion of airway mucus (21), reduce ciliary motility (37), mediate airway smooth muscle contraction (38) and ultimately lead to persistent airflow limitation (39). Siva et al (18) reported that minimization of eosinophilic airway inflammation is associated with a reduction in severe exacerbation of COPD. The investigation of plasma levels of ECP, EDN, EPX and MBP in the present study was therefore required to elucidate the role of eosinophil activation in AECOPD pathogenesis.

In addition to chronic inflammatory disorders, including asthma and COPD, a number of studies have addressed the involvement of eosinophils and their granule proteins in host resistance to parasites, their antimicrobial activities toward bacterial, viral and protozoan pathogens, their function as mediators of hypersensitivity and their interactions with the coagulation system $(22,38)$. Case reports have stated that eosinophilia in patients may be accompanied by thromboembolisms (40-42). The secretion of $\operatorname{ECP}(21,43), \operatorname{EDN}(43)$ and MBP $(43,44)$ has been implicated in increasing the probability of thrombosis formation. MBP also inhibits the binding of thrombomodulin to thrombin and activates protein $\mathrm{C}$, leading to decreased coagulation factor $\mathrm{V}$ degradation (44). ECP, EDN and MBP also damage vascular endothelial cells, expose tissue factors and activate coagulation factor XII to activate the extrinsic coagulation pathway causing hypercoagulability and thrombosis (43); hypereosinophilia may therefore be an important marker in the progression to hypercoagulability. However, no additional increase in the eosinophil count or plasma levels of eosinophil secretory proteins was observed in patients with AECOPD/PE in the present study, suggesting that eosinophils were not further activated during the progression from AECOPD to AECOPD/PE.

ECP and EDN were demonstrated to have high specificity and sensitivity as biomarkers for AECOPD and AECOPD/PE 
A

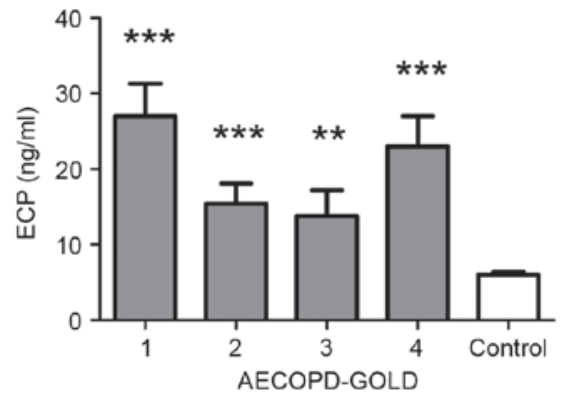

C

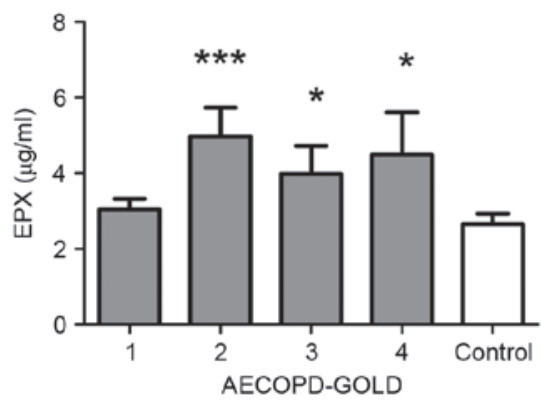

$\mathrm{E}$

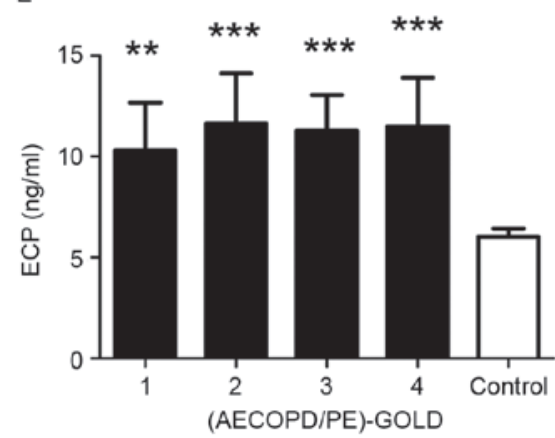

G

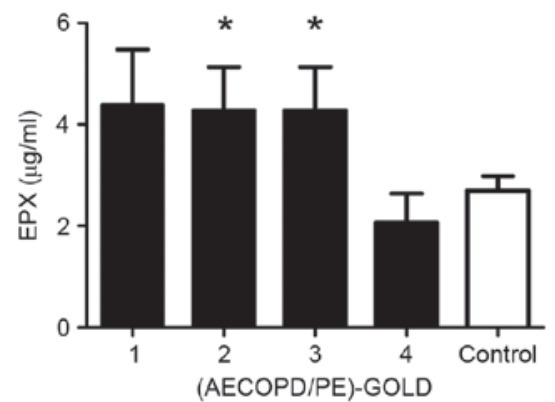

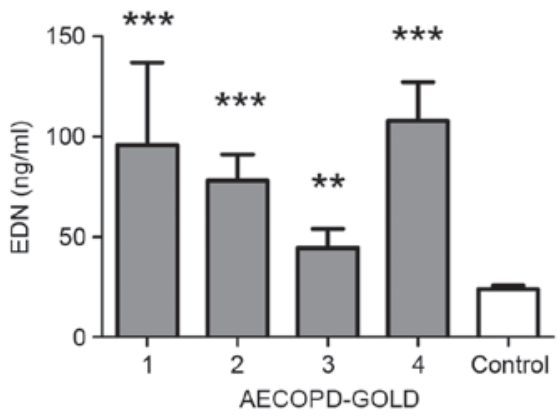

D

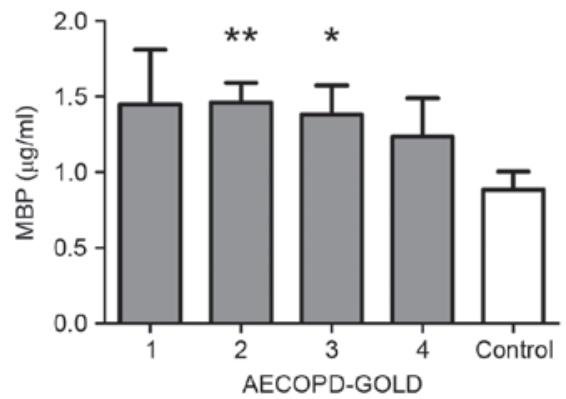

$\mathrm{F}$

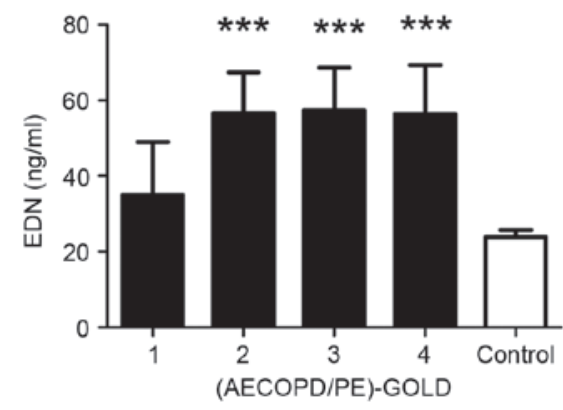

$\mathrm{H}$

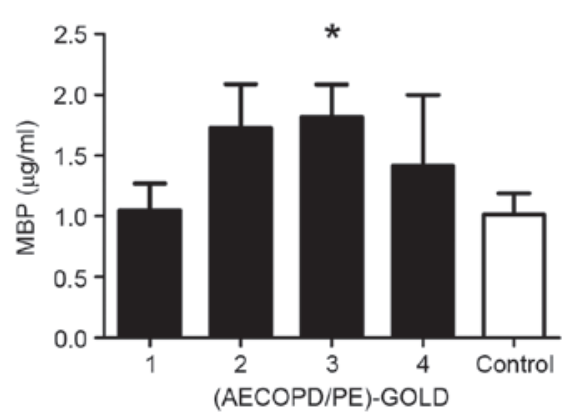

Figure 5. Levels of ECP, EDN, EPX and MBP according to the GOLD index of the groups. Normal controls, $\mathrm{n}=35$. Patients with AECOPD: GOLD1 index, $\mathrm{n}=4$; GOLD2 index, $\mathrm{n}=14$; GOLD3 index, $\mathrm{n}=18$; and GOLD4 index, $\mathrm{n}=11$. Patients with AECOPD/PE: GOLD1 index, $\mathrm{n}=5$; GOLD2 index, $\mathrm{n}=9$; GOLD3 index, $\mathrm{n}=10$; GOLD4 index, $\mathrm{n}=6$. Measurements of $(\mathrm{A}) \mathrm{ECP}\left({ }^{* *} \mathrm{P}<0.01\right.$ and ${ }^{* * * *} \mathrm{P}<0.001$ vs. AECOPD at ECP classified by GOLD-index), (B) EDN $\left({ }^{* *} \mathrm{P}<0.01\right.$ and ${ }^{* * * *} \mathrm{P}<0.001$ vs. AECOPD at EDN classified by GOLD-index), (C) EPX ("P $<0.05$ and ${ }^{* * *} \mathrm{P}<0.001$ vs. AECOPD at EPX classified by GOLD-index) and (D) MBP in patients with AECOPD ("P $<0.05$ and ${ }^{* *} \mathrm{P}<0.01$ vs. AECOPD at MBP classified by GOLD-index). Measurements of (E) ECP $\left({ }^{* *} \mathrm{P}<0.01\right.$ and ${ }^{* * *} \mathrm{P}<0.001 \mathrm{vs}$. AECOPD/PE at ECP classified by GOLD-index), (F) EDN ( ${ }^{* * *} \mathrm{P}<0.001$ vs. AECOPD/PE at EDN classified by GOLD-index), (G) EPX ("P<0.05 vs. AECOPD/ $\mathrm{PE}$ at EPX classified by GOLD-index) and (H) MBP in patients with AECOPD/PE ( $\mathrm{P}<0.05$ vs. AECOPD/PE at MBP classified by GOLD-index. ECP, eosinophil cationic protein; EDN, eosinophil-derived neurotoxin; EPX, eosinophil peroxidase; MBP, major basic protein; GOLD, Global Initiative for Chronic Obstructive Lung Disease; AECOPD, acute exacerbation of chronic obstructive pulmonary disease; PE, pulmonary embolism.

in the present study, whereas MBP had a lower specificity and EPX had a very low sensitivity for predicting AECOPD and
AECOPD/PE. Although the plasma levels of ECP, EDN, EPX and MBP were upregulated in the AECOPD and AECOPD/PE 
groups in the present study, ECP and EDN were the most potent biomarkers for predicting AECOPD and AECOPD/PE. Fiorini et al (24) reported that ECP measured by radioimmunoassay did not increase significantly in patients with stable COPD (24). This suggests that eosinophil activation is not involved in the stable stage of COPD; however, in the acute stage of severe COPD, eosinophil activation appears to be crucial and thus eosinophil secretory proteins, particularly ECP and EDN, are likely to be key clinical indicators for AECOPD.

In addition to patients with AECOPD, patients with AECOPD/PE were assessed for eosinophil activation in the present study. The results indicate that eosinophil secretory proteins are not differentially elevated in the plasma of patients with AECOPD/PE compared with those with AECOPD, suggesting that eosinophil activation is not associated with the progression from AECOPD to AECOPD/PE. These results indicate that eosinophils are activated in the acute stage of COPD and that eosinophil activation may then plateau, even in the event of exacerbation and development of AECOPD/PE.

In conclusion, the results of the present study suggest that critical eosinophil activation occurs during the development of AECOPD, and thus ECP, EDN, MBP and EPX may serve as biomarkers for disease prediction. Of these markers, ECP and EDN appear to be the most robust predictors and merit further investigation. The present study also demonstrated that eosinophils are not further activated in the transition from AECOPD to AECOPD/PE, and so the factors responsible for PE induction in AECOPD remain to be determined.

\section{Acknowledgements}

The present study was supported by an award from Dongyang People's Hospital (Jinhua, China; grant no. 2015-ZD001).

\section{References}

1. Hubbard R: The burden of lung disease. Thorax 61: 557-558, 2006.

2. Vestbo J, Hurd SS and Rodriguez-Roisin R: The 2011 revision of the global strategy for the diagnosis, management and prevention of COPD (GOLD)-why and what? Clin Respir J 6: 208-214, 2012

3. Sapey E and Stockley RA: COPD exacerbations. 2: Aetiology. Thorax 61: 250-258, 2006.

4. Tillie-Leblond I, Marquette CH, Perez T, Scherpereel A, Zanetti C, Tonnel AB and Remy-Jardin M: Pulmonary embolism in patients with unexplained exacerbation of chronic obstructive pulmonary disease: Prevalence and risk factors. Ann Intern Med 144: 390-396, 2006.

5. Choi KJ, Cha SI, Shin KM, Lee J, Hwangbo Y, Yoo SS, Lee J, Lee SY, Kim CH,Park JY and Jung TH: Prevalence and predictors of pulmonary embolism in Korean patients with exacerbation of chronic obstructive pulmonary disease. Respiration 85: 203-209, 2013.

6. Shapira-Rootman M, Beckerman M, Soimu U, Nachtigal A and Zeina AR: The prevalence of pulmonary embolism among patients suffering from acute exacerbations of chronic obstructive pulmonary disease. Emerg Radiol 22: 257-260, 2015.

7. Akpinar EE, Hoşgün D, Akpinar S, Ataç GK, Doğanay B and Gülhan M: Incidence of pulmonary embolism during COPD exacerbation. J Bras Pneumol 40: 38-45, 2014 (In English, Portuguese).

8. Bahloul M, Chaari A, Tounsi A, Baccouche N, Abid H, Chtara K, Ben Hamida C, Ghadhoune H, Dammak H, Chelly H and Bouaziz M: Incidence and impact outcome of pulmonary embolism in critically ill patients with severe exacerbation of chronic obstructive pulmonary diseases. Clin Respir J 9: 270-277, 2015.
9. Groenewegen KH, Schols AM and Wouters EF: Mortality and mortality-related factors after hospitalization for acute exacerbation of COPD. Chest 124: 459-467, 2003.

10. Gunen H, Gulbas G, In E, Yetkin O and Hacievliyagil SS: Venous thromboemboli and exacerbations of COPD. Eur Respir J 35: 1243-1248, 2010.

11. Miszti-Blasius K, Debreceni IB, Felszeghy S, Dezso B and Kappelmayer J: Lack of P-selectin glycoprotein ligand-1 protects mice from thrombosis after collagen/epinephrine challenge. Thromb Res 127: 228-234, 2011.

12. Oelsner EC, Pottinger TD, Burkart KM, Allison M, Buxbaum SG, Hansel NN, Kumar R, Larkin EK, Lange LA, Loehr LR, et al: Adhesion molecules, endothelin-1 and lung function in seven population-based cohorts. Biomarkers 18: 196-203, 2013.

13. Bakakos P, Patentalakis G and Papi A: Vascular biomarkers in asthma and COPD. Curr Top Med Chem 16: 1599-1609, 2016.

14. Poredos P and Jezovnik MK: In patients with idiopathic venous thrombosis, interleukin-10 is decreased and related to endothelial dysfunction. Heart Vessels 26: 596-602, 2011.

15. Liu SF, Kuo HC, Tseng CW, Huang HT, Chen YC, Tseng CC and Lin MC: Leukocyte mitochondrial DNA copy number is associated with chronic obstructive pulmonary disease. PLoS One 10: e0138716, 2015.

16. Tefferi A, Gangat $\mathrm{N}$ and Wolanskyj A: The interaction between leukocytosis and other risk factors for thrombosis in essential thrombocythemia. Blood 109: 4105, 2007.

17. Brightling CE, McKenna S, Hargadon B, Birring S, Green R, Siva R, Berry M, Parker D, Monteiro W, Pavord ID and Bradding P: Sputum eosinophilia and the short term response to inhaled mometasone in chronic obstructive pulmonary disease. Thorax 60: 193-198, 2005.

18. Siva R, Green RH, Brightling CE, Shelley M, Hargadon B, McKenna S, Monteiro W, Berry M, Parker D, Wardlaw AJ and Pavord ID: Eosinophilic airway inflammation and exacerbations of COPD: A randomised controlled trial. Eur Respir J 29: 906-913, 2007.

19. Brightling CE, Monteiro W, Ward R, Parker D, Morgan MD, Wardlaw AJ and Pavord ID: Sputum eosinophilia and short-term response to prednisolone in chronic obstructive pulmonary disease: A randomised controlled trial. Lancet 356: 1480-1485, 2000.

20. Hospers JJ, Schouten JP, Weiss ST, Rijcken B and Postma DS: Asthma attacks with eosinophilia predict mortality from chronic obstructive pulmonary disease in a general population sample. Am J Respir Crit Care Med 160: 1869-1874, 1999.

21. de Oliveira PC, de Lima PO, Oliveira DT and Pereira MC: Eosinophil cationic protein: Overview of biological and genetic features. DNA Cell Biol 31: 1442-1446, 2012.

22. Eriksson J, Woschnagg C, Fernvik E and Venge P: A SELDI-TOF MS study of the genetic and post-translational molecular heterogeneity of eosinophil cationic protein. J Leukoc Biol 82: 1491-1500, 2007.

23. Legrand F, Driss V, Delbeke M, Loiseau S, Hermann E, Dombrowicz D and Capron M: Human eosinophils exert TNF-a and granzyme A-mediated tumoricidal activity toward colon carcinoma cells. J Immunol 185: 7443-7451, 2010.

24. Fiorini G, Crespi S, Rinaldi M, Oberti E, Vigorelli R and Palmieri G: Serum ECP and MPO are increased during exacerbations of chronic bronchitis with airway obstruction. Biomed Pharmacother 54: 274-278, 2000.

25. Shahana S, Kampf C and Roomans GM: Effects of the cationic protein poly-L-arginine on airway epithelial cells in vitro. Mediators Inflamm 11: 141-148, 2002.

26. Vestbo J,Hurd SS, Agustí AG,Jones PW,Vogelmeier C, Anzueto A, Barnes PJ, Fabbri LM, Martinez FJ, Nishimura M, et al: Global strategy for the diagnosis, management, and prevention of chronic obstructive pulmonary disease: GOLD executive summary. Am J Respir Crit Care Med 187: 347-365, 2013.

27. Vaitkus M, Lavinskiene S, Barkauskiene D, Bieksiene K, Jeroch J and Sakalauskas R: Reactive oxygen species in peripheral blood and sputum neutrophils during bacterial and nonbacterial acute exacerbation of chronic obstructive pulmonary disease. Inflammation 36: 1485-1493, 2013.

28. Xiong XZ, Jin Y, Zhou Q, Zhang XJ, Du W, Liu W and Huang SA: Correlation between FoxP3(+) regulatory T cells and chronic obstructive pulmonary disease. Zhonghua Yi Xue Za Zhi 88: 471-474, 2008 (In Chinese).

29. Gutierrez P, Closa D, Piñer R, Bulbena O, Menéndez R and Torres A: Macrophage activation in exacerbated COPD with and without community-acquired pneumonia. Eur Respir J 36: 285-291, 2010. 
30. Chow L, Parulekar AD and Hanania NA: Hospital management of acute exacerbations of chronic obstructive pulmonary disease. J Hosp Med 10: 328-339, 2015

31. Zhu J, Qiu YS, Majumdar S, Gamble E, Matin D, Turato G, Fabbri LM, Barnes N, Saetta M and Jeffery PK: Exacerbations of Bronchitis: Bronchial eosinophilia and gene expression for interleukin-4, interleukin-5 and eosinophil chemoattractants. Am J Respir Crit Care Med 164: 109-116, 2001.

32. Saetta M, Di Stefano A, Maestrelli P, Turato G, Mapp CE, Pieno M, Zanguochi G, Del Prete G and Fabbri LM: Airway eosinophilia and expression of interleukin-5 protein in asthma and in exacerbations of chronic bronchitis. Clin Exp Allergy 26: 766-774, 1996

33. Wever AM, Wever-Hess J and Hermans J: The use of serum eosinophil cationic protein (ECP) in the management of steroid therapy in chronic asthma. Clin Exp Allergy 27: 519-529, 1997.

34. Pesci A, Balbi B, Majori M, Cacciani G, Bertacco S, Alciato P and Donner CF: Inflammatory cells and mediators in bronchial lavage of patients with chronic obstructive pulmonary disease. Eur Respir J 12: 380-386, 1998.

35. Yamamoto C, Yoneda T, Yoshikawa M, Fu A, Tokuyama T, Tsukaguchi $\mathrm{K}$ and Narita N: Airway inflammation in COPD assessed by sputum levels of interleukin-8. Chest 112: 505-510, 1997.

36. Fujimoto K, Yasuo M, Urushibata K, Hanaoka M, Koizumi T and Kubo K: Airway inflammation during stable and acutely exacerbated chronic obstructive pulmonary disease. Eur Respir J 25 : 640-646, 2005.
37. Motojima S, Frigas E, Loegering DA and Gleich GJ: Toxicity of eosinophil cationic proteins for guinea pig tracheal epithelium in vitro. Am Rev Respir Dis 139: 801-805, 1989.

38. Acharya KR and Ackerman SJ: Eosinophil granule proteins: Form and function. J Biol Chem 289: 17406-17415, 2014.

39. Gon Y, Ito R, Hattori T, Hiranuma H, Kumasawa F, Kozu Y, Endo D, Koyama D, Shintani Y, Eriko T, et al: Serum eosinophil-derived neurotoxin: Correlation with persistent airflow limitation in adults with house-dust mite allergic asthma. Allergy Asthma Proc 36: e113-e120, 2015.

40. Xu W, Guo W and Yang T: Hypereosinophilic syndrome with first presentation of pulmonary embolism and extensive venous thrombosis: A case report and literature review. Zhonghua Jie $\mathrm{He}$ He Hu Xi Za Zhi 38: 912-917, 2015 (In Chinese).

41. Ye X, Feng Y and Lin S: Pulmonary embolism as the initial clinical presentation of Kimura disease: Case report and literature review. Blood Coagul Fibrinolysis 26: 414-418, 2015.

42. Ko JH, Lin JW and Hui RC: Acute pulmonary embolism in a patient with hypereosinophilia and psoriasis. Chang Gung Med J 34 (6 Suppl): S17-S23, 2011.

43. Kikuchi K, Minami K, Miyakawa $\mathrm{H}$ and Ishibashi $\mathrm{M}$ : Portal vein thrombosis in hypereosinophilic syndrome. Am J Gastroenterol 97: 1274-1275, 2002

44. Mukai HY, Ninomiya H, Ohtani K, Nagasawa T and Abe T: Major basic protein binding to thrombomodulin potentially contributes to the thrombosis in patients with eosinophilia. $\mathrm{Br}$ J Haematol 90: 892-899, 1995. 